\title{
Betti and Tachibana numbers
}

Sergey E. Stepanov and Josef Mikeř 


\title{
BETTI AND TACHIBANA NUMBERS
}

\author{
SERGEY E. STEPANOV AND JOSEF MIKEŠ
}

\begin{abstract}
We present a rough classification of differential forms on a Riemannian manifold, we consider definitions and properties of conformal Killing forms on a compact Riemannian manifold and define Tachibana numbers as an analog of the well known Betti numbers. We state the conditions that characterize these numbers. In the last section we show connections between the Betti and Tachibana numbers.
\end{abstract}

2000 Mathematics Subject Classification: 53C20, 53C21, 53C24

Keywords: Riemannian manifold, differential form, Betti numbers, Tachibana numbers

\section{INTRODUCTION}

The the paper is based on our plenary lecture at the International Conference AGMP-8 (Brno, 12-14 September 2012). The paper is organized as follows. In the first section, we introduce some notations and give some basic definitions of the theory of differential forms on a Riemannian manifold $(M, g)$ and natural operators on forms. In addition, we present a rough classification of differential forms on a Riemannian manifold. In the next two paragraphs of the paper we consider properties of harmonic and conformal Killing forms on an $n$-dimensional compact Riemannian manifold $(M, g)$ and determine the Tachibana number $t_{r}(M)$ as an analog of the Betti number $b_{r}(M)$ of $(M, g)$ for $1 \leq r \leq n-1$. Moreover, we state some conditions that characterize these numbers. In the last section we formulate results on relationships between Betti and Tachibana numbers.

\section{ON A CLASSIFICATION OF DIFFERENTIAL FORMS ON RIEMANNIAN MANIFOLD}

In this section we will show a rough classification of differential forms on a Riemannian manifold, which will be useful for the establishment of the desired results. Moreover, we want to fix some notations and basic concepts.

Let $(M, g)$ be an $n$-dimensional compact and orientable Riemannian manifold with the Levi-Civita connection $\nabla$ and let $\Omega^{r}(M)$ denote the vector space of smooth

Supported the Czech Science Foundation through Grant P201/11/0356. 
$r$-forms on $M$ for $1 \leq r \leq n-1$. We define an inner product on $\Omega^{r}(M)$ by the formula: $\left\langle\omega, \omega^{\prime}\right\rangle=\int_{M} g\left(\omega, \omega^{\prime}\right) d$ vol for any $\omega, \omega^{\prime} \in \Omega^{r}(M)$. The inner product structures on $\Omega^{r}(M)$ allow us to define the exterior co-differential operator $d^{*}$ : $\Omega^{r}(M) \rightarrow \Omega^{r-1}(M)$ as a formal adjoint to the well known exterior differential operator $d: \Omega^{r}(M) \rightarrow \Omega^{r+1}(M)$ by the following formula $\langle d \omega, \theta\rangle=\left\langle\omega, d^{*} \theta\right\rangle$.

More than thirty years ago, Bourguignon [3] considered the space of Riemannian natural (with respect to isometric diffeomorphisms) first-order differential operators on $\Omega^{r}(M)$ with values in the space of homogeneous tensors on $M$. He proved the existence of a basis of this space which consists of three operators $\left\{D_{1}, D_{2}, D_{3}\right\}$ where $D_{1}:=d$ and $D_{2}:=d^{*}$. As for the third operator $D_{3}$, Bourguignon said that $D_{3}$ does not have any geometric interpretation for $r>1$. It was also pointed out that, in the case $r=1$, the kernel of $D_{3}$ consists of infinitesimal conformal transformations of $M$.

By way of specification of Bourguignon's result, we showed $[14,15]$ that

$$
\begin{aligned}
& D_{1}=\frac{1}{p+1} d, \\
& D_{2}=\frac{1}{n-p+1} g \wedge d^{*}, \\
& D_{3}=\nabla-\frac{1}{p+1} d-\frac{1}{n-p+1} g \wedge d^{*}
\end{aligned}
$$

and proved $[14,15]$ that the kernel of the third basis operator $D_{3}$ consists of conformal Killing $r$-forms. These forms were introduced by Tachibana [22] and Kashiwada [8] as a natural generalization of conformal Killing vector fields, which are also called infinitesimal conformal transformations [12]. The space of conformal Killing $r$-forms will be denoted by $\mathbf{T}^{r}(M, \mathbb{R})$.

In addition, we mention that the kernel of the first basis differential operator $D_{1}$ consists of closed $r$-forms and the kernel of the second basis differential operator $D_{2}$ consists of co-closed $r$-forms. The two vector spaces of closed and co-closed $r$-forms will be denoted by $\mathbf{D}^{r}(M, \mathbb{R})$ and $\mathbf{F}^{r}(M, \mathbb{R})$.

The condition $\omega \in \operatorname{ker} D_{1} \cap \operatorname{ker} D_{2}$ characterizes the form $\omega$ as a harmonic form [11, p. 204], therefore the vector space $\mathbf{H}^{r}(M, \mathbb{R})$ of harmonic $r$-forms is defined as

$$
\mathbf{H}^{r}(M, \mathbb{R})=\mathbf{D}^{r}(M, \mathbb{R}) \cap \mathbf{F}^{r}(M, \mathbb{R}) .
$$

The condition $\omega \in \operatorname{ker} D_{2} \cap \operatorname{ker} D_{3}$ characterizes the $r$-form as a co-closed conformal Killing form, which is also called a Killing form (see [4, p. 65-66] and [16]). Therefore, the vector space $\mathbf{K}^{r}(M, \mathbb{R})$ of co-closed conformal Killing $r$-forms is defined as

$$
\mathbf{K}^{r}(M, \mathbb{R})=\mathbf{T}^{r}(M, \mathbb{R}) \cap \mathbf{F}^{r}(M, \mathbb{R}) .
$$

Co-closed conformal Killing forms can be regarded as a generalization of Killing vector fields that define infinitesimal isometric transformations [12]. 
The condition $\omega \in \operatorname{ker} D_{1} \cap \operatorname{ker} D_{3}$ characterizes $\omega$ as a closed conformal Killing form. Therefore, the space $\mathbf{F}^{r}(M, \mathbb{R})$ of closed conformal Killing $r$-forms is defined as (see [14])

$$
\mathbf{F}^{r}(M, \mathbb{R})=\mathbf{D}^{r}(M, \mathbb{R}) \cap \mathbf{T}^{r}(M, \mathbb{R}) .
$$

Closed conformal Killing forms (which are also called planar forms) are a generalization of closed conformal Killing vector fields, which are also called local gradient infinitesimal conformal transformations [12]. We denote by $\mathbf{C}^{r}(M, \mathbb{R})$ the subspace of $\Omega^{r}(M)$ which consists of parallel $r$-forms, which are also called covariant constant $r$-forms. It is clear that

$$
\mathbf{C}^{r}(M, \mathbb{R})=\mathbf{K}^{r}(M, \mathbb{R}) \cap \mathbf{P}^{r}(M, \mathbb{R}) \subset \mathbf{H}^{r}(M, \mathbb{R}) .
$$

Using definitions and propositions which we have formulated above, we obtain the 3D-diagram of inclusions of subspaces of differential $r$-forms on an $n$-dimensional $(1 \leq r \leq n-1)$ Riemannian manifold $(M, g)$.

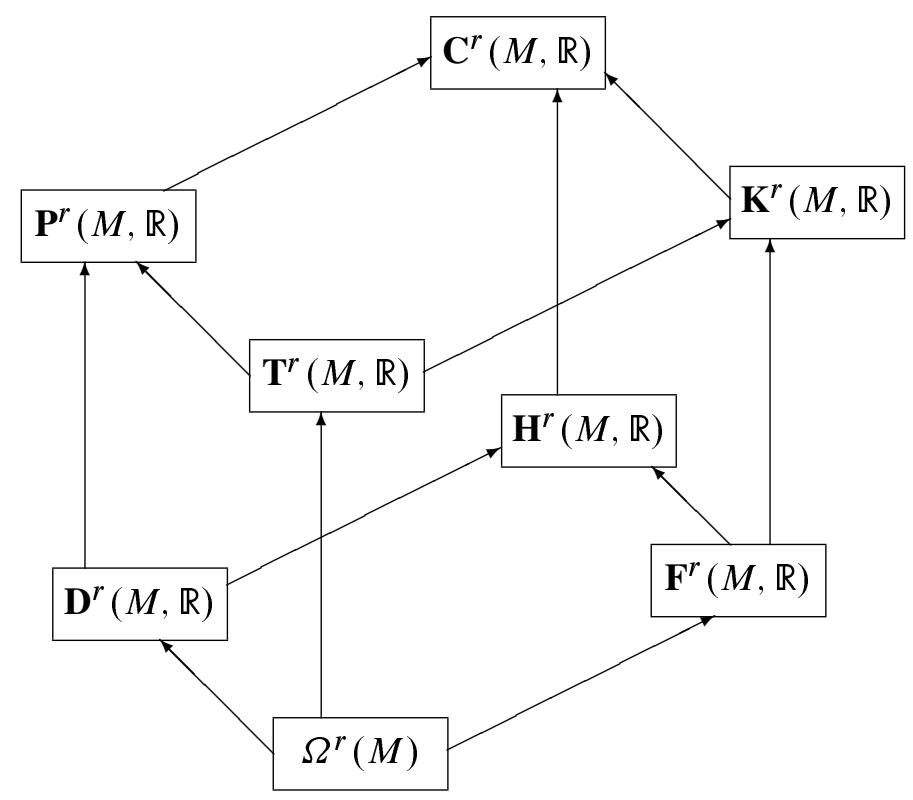

Here, for instance, the arrow $\mathbf{F}^{r}(M, \mathbb{R}) \rightarrow \mathbf{K}^{r}(M, \mathbb{R})$ means that the vector space $\mathbf{K}^{r}(M, \mathbb{R})$ is subspace of $\mathbf{F}^{r}(M, \mathbb{R})$.

\section{HARMONIC FORMS AND BETTI NUMBERS}

One of the motivations for studying Tachibana numbers of a compact Riemannian manifold $(M, g)$ is their close relation to Betti numbers. In this section we will 
present a brief review of the results of the theory of harmonic forms and Betti numbers.

The Laplacian on forms $\Delta: \Omega^{r}(M) \rightarrow \Omega^{r}(M)$, also called the Hodge Laplacian, is defined in terms of the natural Riemannian operators $d$ and $d^{*}$ as $\Delta=d d^{*}+d^{*} d$ (see [3, p. 52], [2, p. 377], [6, p. 316], [11, p. 204]). The operator $\Delta$ is a nonnegative elliptic second order linear differential operator and its kernel is finite dimensional on a compact Riemannian manifold $(M, g)$.

Two following equivalent conditions define $\omega \in \Omega^{r}(M)$ as a harmonic form $d \omega=0$ and $d^{*} \omega=0$ or $\Delta \omega=0$ on a compact Riemannian manifold $(M, g)$ (see [11, p. 204]).

If we denote by $\mathbf{H}^{r}(M, \mathbb{R})$ the vector space of harmonic $r$-forms on a compact Riemannian manifold $(M, g)$, then, by Hodge theory (see [7]), the $r$ th Betti number $b_{r}(M)$ of $(M, g)$ is defined by the equation $b_{r}(M)=\operatorname{dim} \mathbf{H}^{r}(M, \mathbb{R})<\infty$.

The Hodge Laplacian commutes $* \Delta=\Delta *$ with the well known (see [3, p. 33], [11, p. 203]) Hodge star operator $* \Omega^{r}(M) \rightarrow \Omega^{n-r}(M)$, which implies the following isomorphism $* \mathbf{H}^{r}(M, \mathbb{R}) \rightarrow \mathbf{H}^{n-r}(M, \mathbb{R})$. From the isomorphism we obtain (see $\left[11\right.$, p. 389]) the Poincaré duality theorem $b_{r}(M)=b_{n-r}(M)$.

\section{CONFORMAL Killing FORMS AND TACHIBANA NUMBERS}

Before explaining the main results of our paper, we want to point out that they are extensions of our results in [14-20] for conformal Killing forms and Tachibana numbers. In this section we will present a brief review of these results.

Throughout in this section we let $(M, g)$ be an $n$-dimensional compact and oriented Riemannian manifold. For any linear differential operator $D$, the inner product structures on $\Omega^{r}(M)$ allow us to define the formal adjoint operator $D^{*}$ to $D$ (see [3, p. 460]). In the case of the first order natural Riemannian (with respect to isometric diffeomorphisms) operator $D$, we can define a strong Laplacian $D^{*} \circ D$ (see [2, p. 377], [6, p. 316-317]). The first simple property of these operators comes from the fact that any strong Laplacian is a non-negative elliptic second order linear differential operator (see [6, p. 314-315]) with a finite dimensional kernel (see [3, pp. 461-463]). For the other properties of these operators, see [2,6, 11,24].

We showed in [17] that the formal adjoint operator $D_{3}^{*}$ to $D_{3}$ determined by the formula $D_{3}^{*}=\nabla^{*}-\frac{1}{p+1} d^{*}-\frac{1}{n-p+1} d \circ$ trace and the Tachibana Laplacian $\square=D_{3}^{*} \circ D_{3}$ have the form

$$
\square=D_{3}^{*} \circ D_{3}=\frac{1}{r(r+1)}\left(\bar{\Delta}-\frac{1}{r+1} d^{*} \circ d-\frac{1}{n-r+1} d \circ d^{*}\right) .
$$

The symbol $\bar{\Delta}$ is called the Bochner rough Laplacian and $\bar{\Delta}:=\nabla^{*} \circ \nabla$ where we denote the formal adjoint of $\nabla$ by $\nabla^{*}$ (see [3, p. 52], [2, p. 377]). 
We proved the following three propositions (see [17]):

$$
\begin{aligned}
& \omega \in \mathbf{T}^{r}(M, \mathbb{R}) \Leftrightarrow \omega \in \operatorname{ker}\left(D_{3}^{*} D_{3}\right) ; \\
& \omega \in \mathbf{K}^{r}(M, \mathbb{R}) \Leftrightarrow \omega \in \operatorname{ker}\left(D_{3}^{*} D_{3}\right) \cap \operatorname{ker} d^{*} ; \\
& \omega \in \mathbf{P}^{r}(M, \mathbb{R}) \Leftrightarrow \omega \in \operatorname{ker}\left(D_{3}^{*} D_{3}\right) \cap \operatorname{ker} d .
\end{aligned}
$$

It is known that the kernel of the Tachibana Laplacian $\square=D_{3}^{*} \circ D_{3}$ has a finite dimension and, therefore, we concluded that (see [20])

$$
\begin{aligned}
& \operatorname{dim} \mathbf{T}^{r}(M, \mathbb{R})=\operatorname{dim}_{\mathbb{R}}\left(\operatorname{ker} D_{3}^{*} D_{3}\right)=t_{r}(M)<\infty ; \\
& \operatorname{dim} \mathbf{K}^{r}(M, \mathbb{R})=k_{r}(M)<\infty ; \\
& \operatorname{dim} \mathbf{P}^{r}(M, \mathbb{R})=p_{r}(M)<\infty
\end{aligned}
$$

on a compact Riemannian manifold $(M, g)$. The numbers $t_{r}(M), k_{r}(M)$ and $p_{r}(M)$ we have called the Tachibana number, the Killing number and the planarity number of a compact Riemannian manifold $(M, g)$, respectively (see [18]). These numbers satisfy the following duality properties $t_{r}(M)=t_{n-r}(M)$ and $p_{r}(M)=k_{n-r}(M)$ for all $r=1, \ldots, n-1$. These equalities are analogues of the Poincare duality for Betti numbers and corollaries of the following isomorphisms (see $[9,14,15])$

$$
*: \mathbf{T}^{r}(M, \mathbb{R}) \rightarrow \mathbf{T}^{n-r}(M, \mathbb{R}) \text { and } *: \mathbf{P}^{r}(M, \mathbb{R}) \rightarrow \mathbf{K}^{n-r}(M, \mathbb{R}) .
$$

Moreover, Tachibana numbers $t_{r}(M)$ are conformal scalar invariants, the Killing number $k_{r}(M)$ and the planarity number $p_{r}(M)$ are projective scalar invariants of a Riemannian manifold $(M, g)$ for all $r=1, \ldots, n-1$. In addition, we mention that the first proposition is a corollary of conformal invariance of conformal Killing $r$-forms (see [1]). The second proposition of our theorem is a corollary of projective invariance of closed and co-closed conformal Killing $r$-forms (see [16]).

Let $(M, g)$ be an $n$-dimensional connected Riemannian manifold. Then the Tachibana number $t_{r}(M)$, the Killing number $k_{r}(M)$ and the planarity number $p_{r}(M)$ of $(M, g)$ satisfy the following inequalities

$$
\begin{gathered}
0 \leq t_{r}(M) \leq \frac{(n+2) !}{(r+1) !(n-r+1) !} ; \quad 0 \leq k_{r}(M) \leq \frac{(n+1) !}{(r+1) !(n-r) !} \\
0 \leq p_{r}(M) \leq \frac{(n+1) !}{r !(n-r+1) !} ;
\end{gathered}
$$

for all $r=1, \ldots, n-1$. Moreover, any of two numbers $p_{r}(M)$ and $k_{r}(M)$ is maximal if and only if $(M, g)$ is a Riemannian manifold with positive constant curvature (see $[10,14,16])$. In addition, the Tachibana number $t_{r}(M)$ is maximal if and only if $(M, g)$ is conformal flat Riemannian manifold (see $[8,13])$.

\section{TACHIBANA AND BETTI NUMBERS}

In this section we will show some relationships between Tachibana and Betti numbers. 
First, we formulate the vanishing theorems of Betti and Tachibana numbers. Let $(M, g)$ be an $n$-dimensional compact oriented Riemannian manifold and let $\mathfrak{R}: \Omega^{2}(M) \rightarrow \Omega^{2}(M)$ be the standard symmetric Riemannian curvature operator of $(M, g)$ (see [11, p. 35-36]).

Assuming $\Re \geq 0$, we have $b_{r}(M) \leq \frac{n !}{r !(n-r) !}=b_{r}\left(T^{n}\right)$ where $T_{n}$ is a flat Riemannian $n$-torus and $r=1, \ldots, n-1$ (see [11, p. 212]). Moreover, an $n$ dimensional compact Riemannian manifold with the positive curvature operator $\mathfrak{R}$ is a spherical space form (see [5]) and its Betti numbers $b_{1}(M), \ldots, b_{n-1}(M)$ are zeros (see [11, p. 212]). In addition, we proved (see [20]) that, in this case, an arbitrary conformal Killing $r$-form $\omega$ is uniquely decomposed in the form $\omega^{\prime}+\omega^{\prime \prime}$ where $\omega^{\prime}$ is a Killing and $\omega^{\prime \prime}$ is a closed conformal Killing $r$-forms on $(M, g)$ for all $r=1, \ldots, n-1$ and hence $t_{r}(M)=k_{r}(M)+p_{r}(M)$.

On the other hand, if $\Re$ is non-positive, then $t_{r}(M) \leq \frac{n !}{r !(n-r) !}=t_{r}\left(T^{n}\right)$ and, if $\Re<0$ somewhere, then $t_{r}(M)=0$ for $r=1, \ldots, n-1$ (see [20]).

Second, we consider Betti and Tachibana numbers of a compact conformally flat Riemannian manifold and prove the following theorem as a corollary of above results.

Theorem 1. Let $(M, g)$ be an $n$-dimensional $(n \geq 3)$ compact conformally flat Riemannian manifold with the positive or negative definite Ricci tensor Ric then $t_{r}(M) \cdot b_{h}(M)=0$ for all $h, r=1, \ldots, n-1$.

Proof. Let one the two following conditions be satisfied Ric $>0$ or Ric $<0$ at every point of $(M, g)$. If we suppose that $(M, g)$ is conformally flat Riemannian manifold of dimension $n \geq 3$, then, in the first case, we have $\mathfrak{R}>0$ and Betti numbers $b_{1}(M)=\cdots=b_{n-1}(M)=0$ because there are no non-zero harmonic $r$-forms for all $r=1, \ldots, n-1$ (see [4, pp. 78-79]). On the other hand, in the second case, we have $\mathfrak{R}<0$ and Tachibana numbers $t_{1}(M)=\cdots=t_{n-1}(M)=0$ because there are no non-zero conformal Killing $r$-forms for all $r=1, \ldots, n-1$ (see [19]). Therefore, we conclude that $t_{r}(M) \cdot b_{h}(M)=0$ for all $h, r=1, \ldots, n-1$, which finishes the proof of the theorem.

Next, we consider the case of an even-dimensional compact and oriented conformally flat Riemannian manifold. In this special case, the following theorem is true.

Theorem 2. Let $(M, g)$ be a $2 r$-dimensional compact and oriented conformally flat Riemannian manifold. Then, the following propositions are true.

(1) If the scalar curvature $s \neq 0$ and the Tachibana number $t_{r}(M) \neq 0$, then the Betti number $b_{r}(M)=0$. Moreover, if $s$ is a positive constant, then

$$
t_{r}(M)=k_{r}(M)+p_{r}(M)
$$

for $k_{r}(M)=\operatorname{dim}_{\mathbb{R}}\left(\operatorname{ker} D_{3}^{*} D_{3} \cap \operatorname{Im} d^{*}\right)$ and $p_{r}(M)=\operatorname{dim}_{\mathbb{R}}\left(\operatorname{ker} D_{3}^{*} D_{3} \cap\right.$ $\operatorname{Im} d)$.

(2) If the scalar curvature $s \neq 0$ and the Betti number $b_{r}(M) \neq 0$, then the Tachibana number $t_{r}(M)=0$. 
(3) If the scalar curvature $s=0$, then the Betti number $b_{r}(M) \neq 0$ if and only if the Tachibana number $t_{r}(M) \neq 0$ and, moreover,

$$
t_{r}(M)=b_{r}(M)=(2 r) !(r !)^{-2} .
$$

Proof. First of all, we recall, that the Hodge Laplacian operator $\Delta$ admits the well known Weitzenböck decomposition (see [3, p. 53])

$$
\Delta \omega=\bar{\Delta} \omega+F_{r}(\omega)
$$

where (see [4, pp. 60-61])

$$
\begin{gathered}
F_{r}(\omega)\left(X_{1}, \ldots, X_{r}\right)=\sum_{\alpha=1}^{r} \operatorname{Ric}\left(e_{j}, X_{\alpha}\right) \omega\left(X_{1}, \ldots, X_{\alpha-1}, e_{j}, X_{\alpha+1}, \ldots, X_{r}\right) \\
-\sum_{\alpha<\beta}^{1, \ldots, r} R\left(e_{j}, e_{k}, X_{\alpha}, X_{\beta}\right) \omega\left(X_{1}, \ldots, X_{\alpha-1}, e_{j}, X_{\alpha+1}, \ldots, X_{\beta-1}, e_{k}, X_{\beta+1}, \ldots, X_{r}\right)
\end{gathered}
$$

for an arbitrary $\omega \in \Omega^{r}(M) ; X_{1}, \ldots, X_{r} \in C^{\infty} T M$, for any orthonormal basis $\left\{e_{1}, \ldots, e_{n}\right\}$, and the curvature tensor $R$ and the Ricci tensor Ric of $(M, g)$. As a consequence of this fact, we obtain from (4.1) the following

$$
\square \omega=\frac{1}{r(r+1)}\left(\Delta \omega-F_{r}(\omega)-\frac{1}{r+1} d^{*} d \omega-\frac{1}{n-r+1} d d^{*} \omega\right)
$$

for any $\omega \in \Omega^{r}(M)$. In particular, we have

$$
\Delta \omega=F_{r}(\omega)+\frac{1}{r+1} d^{*} d \omega+\frac{1}{n-r+1} d d^{*} \omega
$$

for an arbitrary conformal Killing $r$-form $\omega$.

Next, if we suppose that our Riemannian manifold $(M, g)$ has the dimension $2 r$ and can be reduced to an Euclidean space by suitable conformal transformation the metric tensor $g$, then we have the equality (see [4, p. 79])

$$
F_{r}(\omega)=\frac{1}{2(2 r-1)} s \cdot \omega,
$$

After that, from (5.3) and (5.4) we can deduce the equation

$$
\Delta \omega=\frac{r+1}{2 r(2 r-1)} s \cdot \omega,
$$

where $s$ is the scalar curvature of $(M, g)$. In particular, if $s \neq 0$ we deduce from (5.5) that $\Delta \omega=0$ if and only if $\omega=0$. Moreover, if we suppose that $s$ is a positive constant, we can rewrite the equation (5.5) in the form

$$
\omega=d^{*} d \omega^{\prime}+d d^{*} \omega^{\prime}
$$


where $\omega^{\prime}=\frac{2 r(2 r-1)}{(r+1) s} \omega$. The equality (5.6) is the well known Hodge - de Ram decomposition where there is no harmonic form. From (5.6) we conclude the following orthogonal decomposition

$$
\mathbf{T}^{r}(M, \mathbb{R})=\mathbf{P}^{r}(M, \mathbb{R}) \oplus \mathbf{K}^{r}(M, \mathbb{R})
$$

where

$$
\mathbf{K}^{r}(M, \mathbb{R})=\left\{\omega \in \Omega^{r}(M) \mid \omega \in \operatorname{ker} D_{3}^{*} D_{3} \cap \operatorname{Im} d^{*}\right\}
$$

and

$$
\mathbf{P}^{r}(M, \mathbb{R})=\left\{\omega \in \Omega^{r}(M) \mid \omega \in \operatorname{ker} D_{3}^{*} D_{3} \cap \operatorname{Im} d\right\}
$$

From (5.7) - (5.9), we obtain the following equalities $t_{r}(M)=k_{r}(M)+p_{r}(M)$, $k_{r}(M)=\operatorname{dim}_{\mathbb{R}}\left(\operatorname{ker} D_{3}^{*} D_{3} \cap \operatorname{Im} d^{*}\right)$ and $p_{r}(M)=\operatorname{dim}_{\mathbb{R}}\left(\operatorname{ker} D_{3}^{*} D_{3} \cap \operatorname{Im} d\right)$, which finish the proof of the first assertion of the theorem.

Next, from (4.1), (5.1) and (5.4) we infer

$$
\frac{s}{2(2 r-1)} \omega=\frac{r}{r+1} \Delta \omega-r(r+1) \square \omega
$$

for any $\omega \in \Omega^{r}(M)$.

Then, for an arbitrary harmonic $r$-form $\omega$ we have

$$
\frac{s}{2(2 r-1)} \omega=-r(r+1) \square \omega .
$$

In particular, if $s \neq 0$ we deduce from (5.5) that $\square \omega=0$ if and only if $\omega=0$.

Finally, we consider a $2 r$-dimensional compact conformally flat Riemannian manifold with zero scalar curvature. In this case, from (5.10) we obtain $\Delta \omega=(r+$ $1)^{2} \square \omega$. If we suppose that $b_{r}(M) \neq 0$, then there exists a nonzero harmonic $r$ form $\omega$ such that $\Delta \omega=(r+1)^{2} \square \omega=0$. Then, $\omega$ is conformal Killing and from (5.1) we obtain the equation $\nabla \omega=0$. In this case we have $b_{r}(M)=t_{r}(M)=$ $\frac{n !}{r !(n-r) !}=(2 r) ! \cdot(r !)^{-2}$, because a parallel form is completely determined by its value at the point. This finishes the proof of the theorem.

As a consequence of the previous theorem we obtain the following corollary.

Corollary 1. Let $(M, g)$ be a $2 r$-dimensional compact and oriented conformally flat Riemannian manifold with constant nonzero scalar curvature s. Then, any nonzero conformal Killing $r$-form is an eigenform of the Hodge Laplacian $\Delta$ corresponding to the eigen-value $\lambda=(r+1)(2 r(2 r-1))^{-1} s$ for $s>0$ and any nonzero harmonic $r$-form is an eigenform of the Tachibana Laplacian $\square$ corresponding to the eigenvalue $\lambda=-\left((2 r(r+1)(2 r-1))^{-1} s\right.$ for $s<0$.

Proof. It is well known that, if a nonzero $r$-form $\omega$ satisfies the equation $\Delta \omega=$ $\lambda \omega$ for a constant $\lambda>0$, it is called an eigenform of the Laplacian $\Delta$ corresponding to the eigen-value $\lambda$. Then, if we suppose that $s$ is a positive constant, then, from (5.5), we conclude that the conformal Killing $r$-form $\omega$ is an eigenform of $\Delta$ corresponding to the eigenvalue $\lambda=(r+1)(2 r(2 r-1))^{-1} s$ (see also [21]). 
Moreover, if we suppose that $\mathrm{s}$ is a negative constant, then, from (5.11), we conclude that the harmonic $r$-form $\omega$ is an eigenform of $\square$ corresponding to the eigenvalue $\lambda=-\left((2 r(r+1)(2 r-1))^{-1} s\right.$.

Next, we consider conformal Killing and harmonic forms on a Riemannian manifold of constant sectional curvature, which is an example of a conformally flat Riemannian manifold.

Theorem 3. Let $(M, g)$ be an $n$-dimensional compact and oriented Riemannian manifold of constant nonzero sectional curvature $C$. Then, we have three propositions.

(1) A nonzero $r$-form $\omega$ is harmonic if and only if $\omega$ is an eigenform of the Tachibana Laplacian $\square$ corresponding to the eigenvalue $\lambda=-(n-r)(r+$ 1) ${ }^{-1} C$ for $C<0$.

(2) Any nonzero closed conformal Killing $r$-form $\omega$ is an eigenform of the Hodge Laplacian $\Delta$ corresponding to the eigenvalue $\lambda=r(n-r+1) C$ for $C>0$. For $n<2 r$ converse is true.

(3) Any nonzero Killing $r$-form $\omega$ is an eigenform of the Hodge Laplacian $\Delta$ corresponding to the eigenvalue $\lambda=(n-r)(r+1) C$ for $C>0$. For $n>2 r$ converse is true.

Proof. Let $(M, g)$ be an $n$-dimensional compact and oriented Riemannian manifold with constant sectional curvature $C$. Then (see [9]), we have the identity

$$
F_{r}(\omega)=r(n-r) C \cdot \omega
$$

for an arbitrary $\omega \in \Omega^{r}(M)$. In this case we can rewrite (5.2) in the form

$\square \omega=\frac{1}{r(r+1)}\left(\Delta \omega-r(n-r) C \cdot \omega-\frac{1}{r+1} d^{*} d \omega-\frac{1}{n-r+1} d d^{*} \omega\right)$.

From this, we see $d \omega=0$ and $d^{*} \omega=0$ hold if and only if

$$
\square \omega=(n-r)(r+1)^{-1} C \cdot \omega
$$

holds. On the other hand, we can rewrite (5.2) in two different forms

$$
\square \omega=\frac{1}{r(r+1)}\left(\frac{n-r}{n-r+1} \Delta \omega-r(n-r) C \cdot \omega-\frac{n-2 r}{(r+1)(n-r+1)} d^{*} d \omega\right)
$$

and

$$
\square \omega=\frac{1}{r(r+1)}\left(\frac{r}{r+1} \Delta \omega-r(n-r) C \cdot \omega+\frac{n-2 r}{(r+1)(n-r+1)} d d^{*} \omega\right) .
$$

From (5.14) we can conclude that if $d \omega=0$ and $\square \omega=0$ hold, then $\Delta \omega=r(n-$ $r+1) C \cdot \omega$ holds. At the same time, from (5.15) we conclude that if $d^{*} \omega=0$ and $\square \omega=0$ hold, then $\Delta \omega=(n-r)(r+1) C \cdot \omega$ holds. It is easy to see that converses are true only for cases when $n<2 r$ and $n>2 r$, respectively. 
In the following theorem, we study compact Riemannian manifolds whose Ricci tensor is negative and positive semi-definite and we give the lower bound for the Betti number $b_{r}(M)$ and the Tachibana number $t_{r}(M)$, respectively. More precisely, we have

Theorem 4. Let $(M, g)$ be a compact n-dimensional Riemannian manifold satisfy one of the following conditions:

(1) the Ricci tensor Ric of $(M, g)$ is negative semi-define and the first Tachibana number $t_{1}(M)=h \leq n$;

(2) the Ricci tensor Ric of $(M, g)$ is positive semi-define and the Betti number $b_{1}(M)=h \leq n$. Then,

$$
\frac{h !}{r !(h-r)} \leq b_{r}(M)=t_{r}(M) \leq \frac{n !}{r !(h-r)}
$$

for $1 \leq r<h=b_{1}(M) \leq n$. If Ric $\leq 0$ and $t_{1}(M)=n$ or Ric $\geq 0$ and $b_{1}(M)=n$, then $(M, g)$ is a flat Riemannian $n$-torus.

Proof. Let $(M, g)$ be a compact $n$-dimensional Riemannian manifold and $t_{1}(M)=$ $h \neq 0$. Then there are $h$ linearly independent nonzero conformal Killing 1-forms $\omega_{1}, \ldots, \omega_{h}$. We denote by $X_{1}, \ldots, X_{h}$ the dual conformal Killing vector fields, i. e. $\omega_{a}(Y)=g\left(Y, X_{a}\right)$ for $a=1, \ldots, h$. If we suppose that the Ricci tensor Ric is negative semi-definite, then $X_{1}, \ldots, X_{h}$ are parallel (see [4, p. 53-55]), i.e. $\nabla X_{1}=0, \ldots, \nabla X_{h}=0$. In this case, the following 1-forms $\omega_{1}, \ldots, \omega_{h}$ are parallel and, hence, are harmonic 1-forms. Using this result, we conclude that $\theta_{i_{1} i_{2} \cdots i_{r}}=$ $\omega_{i_{1}} \wedge \omega_{i_{2}} \wedge \cdots \wedge \omega_{i_{r}}$ for any $1 \leq i_{1}<\cdots<i_{r} \leq h$ are parallel $r$-forms and, hence, conformal Killing $r$-forms. Since these $\theta_{i_{1} i_{2} \cdots i_{r}}$ are $\frac{h !}{r !(h-r) !}$ linearly independent conformal Killing $r$-forms, we have $t_{r}(M) \geq \frac{h !}{r !(h-r) !}$ for $r<h$. Moreover, we have $b_{r}(M) \geq \frac{h !}{r !(h-r) !}$ for $r<h$ because an arbitrary parallel $r$-form $\omega$ is harmonic. On the other hand, let $(M, g)$ be a compact $n$-dimensional Riemannian manifold with $b_{1}(M)=h \neq 0$. Then, there are $h$ linearly independent nonzero harmonic 1 -forms $\omega_{1}, \ldots, \omega_{h}$. We denote by $X_{1}, \ldots, X_{h}$ the dual harmonic vector fields, i. e. $\omega_{a}(Y)=g\left(Y, X_{a}\right)$ for $a=1, \ldots, h$. If we suppose that the Ricci tensor Ric is positive semi-definite, then $X_{1}, \ldots, X_{h}$ are parallel (see [4, p. 53-55]), i.e. $\nabla X_{1}=0, \ldots, \nabla X_{h}=0$. From this we can conclude that $b_{r}(M) \geq \frac{h !}{r !(h-r) !}$ for $r<h$ and hence $t_{r}(M) \geq \frac{h !}{r !(h-r) !}$. Next, if Ric $\leq 0$ and $t_{1}(M)=n$ or Ric $\geq 0$ and $b_{1}(M)=n$, then $(M, g)$ has a parallel frame. The curvature tensor of $(M, g)$ vanishes in this frame, so $(M, g)$ is a flat torus (see [11, p. 208]) and hence $b_{r}(M)=t_{r}(M)=\frac{h !}{r !(h-r) !}$. This concludes the proof of the theorem.

Finally, we prove the following theorem and formulate its corollary. 
Theorem 5. Let $(M, g)$ be a compact $n$-dimensional Riemannian manifold with its zero first Betti number $b_{1}(M)$ and non-zero first planarity number $p_{1}(M)$. Then, Betti numbers $b_{2}(M), \ldots, b_{n-1}(M)$ equal zeros, too.

Proof. If $b_{1}(M)=0$ and $p_{1}(M) \neq 0$, then there exists a non-zero exact conformal Killing 1-form $\omega=d f$ such that $\nabla \operatorname{grad} f=-n^{-1} \Delta f \cdot g$ for a smooth function $f$. In this case, due to Tashiro theorem (see [23]), $(M, g)$ is conformally diffeomorphic to a Euclidean sphere $\mathbb{S}^{n}$ and hence $b_{r}(M)=b_{r}\left(\mathbb{S}^{n}\right)=0$ for $r=1, \ldots, n-1$. This finishes the proof.

Corollary 2. Let $(M, g)$ be a compact $n$-dimensional Riemannian manifold such that its Betti number $b_{n-1}(M)=0$ and Killing number $k_{n-1}(M) \neq 0$. Then, Betti numbers $b_{1}(M), \ldots, b_{n-2}(M)$ equal zeros, too.

Acknowledgment. The paper was supported by grant P201/11/0356 of the Czech Science Foundation.

\section{REFERENCES}

[1] I. M. Benn and P. Charlton, "Dirac symmetry operators from conformal Killing-Yano tensor," Classical Quantum Gravity, vol. 14, pp. 1037-1042, 1997.

[2] P. H. Berard, "From vanishing theorems to estimating theorems: the Bochner technique revisited," Bull. Am. Math. Soc., vol. 19, pp. 371-406, 1988.

[3] A. L. Besse, Einstein manifolds. Berlin-Heidelberg: Springer-Verlag, 1987.

[4] S. Bochner and K. Yano, Curvature and Betti numbers. Princeton Univ. Press, 1953.

[5] C. Böhm and B. Wilkig, "Manifolds with positive curvature operators are space forms," Ann. Math., vol. 167, pp. 1079-1097, 2008.

[6] J. P. Bourguignon, "Formules de Weitzenbök en dimension 4," Semin. Arthur Besse, Paris 1978/79, pp. 308-333, 1981.

[7] G. de Rham, Differentiable manifolds. Berlin-Heidelberg: Springer-Verlag, 1984.

[8] T. Kashiwada, "On conformal killing tensor," Natur. Sci. Rep. Ochanomizu Univ., vol. 19, pp. 67-74, 1968.

[9] M. Kora, "On conformal Killing forms and the proper space of $\Delta$ for p-forms," Math. J. Okayama Univ., vol. 22, pp. 195-204, 1980.

[10] J. Mikeš, I. Hinterleitner, and S. E. Stepanov, "Projective mappings and dimensions of vector spaces of three types of Killing-Yano tensors on pseudo-Riemannian manifolds of constant curvature," AIP Conf. Proc., vol. 1460, pp. 202-205, 2012.

[11] P. Petersen, Riemannian Geometry. Springer, 2006.

[12] H. B. Rademacher and W. Kühnel, "Conformal transformations of pseudo-Riemannian manifolds," in Recent developments in Pseudo-Riemannian geometry. Zürich: EMS Publ. House, 2008, pp. 261-298.

[13] U. Semmelmann, "Conformal Killing forms on Riemannian manifolds," Math. Z., vol. 245, pp. 503-527, 2003.

[14] S. E. Stepanov, "A class of closed forms and special Maxwell's equations," Tensor, N.S., vol. 58, pp. 233-242, 1997.

[15] S. E. Stepanov, "On conformal Killing 2-form of the electromagnetic field," J. Geom. Phys., vol. 33, pp. 191-209, 2000.

[16] S. E. Stepanov, “The Killing-Yano tensor,” Theor. Math. Phys., vol. 134, pp. 333-338, 2003. 
[17] S. E. Stepanov, "A new strong Laplacian on differential forms," Math. Notes, vol. 76, pp. 420-425, 2004.

[18] S. E. Stepanov, "Some conformal and projective scalar invariants of Riemannian manifolds," Math. Notes, vol. 80, pp. 848-852, 2006.

[19] S. E. Stepanov, "Vanishing theorem in affine, Riemannian and Lorentz geometries," J. Math. Sci., vol. 141, pp. 929-964, 2007.

[20] S. E. Stepanov, "Curvature and Tachibana numbers," Sb. Math., vol. 202, pp. 135-146, 2011.

[21] S. Tachibana, "On the proper space of $\Delta$ for m-forms in $2 \mathrm{~m}$-dimensional conformal flat Riemannian manifolds," Nat. Sci. Rep. Ochanomizu Univ., vol. 28, pp. 111-115, 1978.

[22] S. Tachibana, "On conformal Killing tensor in a Riemannian space," Tohoku Math. J., vol. 21, pp. 56-64, 1969.

[23] Y. Tashiro, "Complete Riemannian manifolds and some vector fields," Trans. Am. Math. Soc., vol. 117, pp. 251-275, 1965.

[24] H. H. Wu, The Bochner technique in differential geometry. London: Academic Publ., 1988.

\section{Authors' addresses}

Sergey E. Stepanov

Finance University under the Government of Russian Federation, Dept. of Mathematics, Leningradsky Prospect, 49-55, 125993, Moscow, Russia

E-mail address: s.s.stepanova@mail.ru

\section{Josef Mikeš}

Palacky University, Faculty of Science, Dept. of Algebra and Geometry, 17. listopadu 12, 77146, Olomouc, Czech Republic

E-mail address: josef.mikes@upol.cz 